\title{
MTA Cvek Pulpotomy Followed by Fragment Re-Attachment in Traumatized Young Permanent Maxillary Left Central Incisor - A Case Report
}

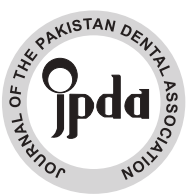

\author{
Abul Khair Zalan ${ }^{1}$ \\ BDS \\ Khadeejah Khalil Zubairy ${ }^{2}$ \\ BDS \\ Hira Zaman ${ }^{3}$ \\ BDS, MDS \\ Anser Maxood ${ }^{4}$ \\ BDS, FRACDS, FICD, MSc \\ Anika Gul ${ }^{5}$ \\ BDS \\ Miraat Anser $^{6}$ \\ BDS
}

Most common form of dental trauma in children are the fractures of crown mainly in anterior teeth. A case of complicated crown fracture treated with Cvek pulpotomy using MTA followed by fragment re-attachment with careful follow-up is presented here. Tooth fragment re-attachment provides feasible conservative alternative approach to restore esthetics and tooth function. Successful outcomes have been shown by the long term follow up of the treatment with the preservation of pulp vitality and continued development of root. Clinical results have also shown good esthetics and functional results of presented technique. KEYWORDS: Complicated crown fracture, Cvek pulpotomy, MTA, fragment re-attachment

HOW TO CITE: Zalan AK, Zubairy KK, Zaman H, Maxood A, Gul A, Anser M. MTA cvek pulpotomy followed by fragment re-attachment in traumatized young permanent maxillary left central incisor - A case report. J Pak Dent Assoc 2021;30(1):70-73. DOI: https://doi.org/10.25301/JPDA.301.70

Received: 24 July 2020, Accepted: 18 November 2020

\section{INTRODUCTION}

$\mathrm{M}$ ost common injuries in children and young adults are the Traumatic dental injuries (TDIs). Among all the dental injuries, luxations and crown fractures occurs more frequently. Maxillary Central incisor $80 \%$ followed by maxillary lateral incisor $20 \%$ are the commonly affected teeth because of their position in the arch. ${ }^{1}$

According to Andreason's classification, Dental fractures involving enamel, or both dentin and enamel but without puplal exposure are termed as uncomplicated crown fractures. But when the fracture is associated with pulp exposure, it is classified as a complicated crown fracture and an endodontic

1. MDS (Resident), Post graduate, Department of Pediatric Dentistry, Pakistan Institute of Medical Sciences.

2. House officer, Department of Pediatric Dentistry, Pakistan Institute of Medical Sciences.

3. Registrar, Department of Operative Dentistry, University College of Dentistry.

4. Dean of Dentistry, Department of Operative \& Pediatric Dentistry, Pakistan Institute of Medical Sciences.

5. FCPS (Resident), Post graduate, Department of Orthodontics, Sardar Begum Dental College.

6. MDS (Resident), Post graduate, Department of Pediatric Dentistry, Pakistan Institute of Medical Sciences.

Corresponding author: “Dr. Abul Khair Zalan” < zalanjan@yahoo.com > treatment should be considered before the definitive treatment. Approximately $18-20 \%$ of all traumatic injuries to permanent teeth are complicated crown fractures. ${ }^{2}$

Management of coronal tooth fractures depends on multiple factors which include extent and pattern of fracture, involvement of pulp tissue, violation of biological width, fracture of alveolus, any associated soft tissue injury and presence or absence of fractured tooth segment. ${ }^{3}$

In young permanent teeth, the treatment of crown fractures with pulp exposure highly depends on extent of pulp tissue involvement, the degree of development of root and most importantly the time period between the examination and traumatic incident. ${ }^{3}$ Pulp should be preserved whenever possible in such teeth with immature roots to allow root formation and tooth maturation. Pulp capping is recommended when size of pulpal exposure is less then $1 \mathrm{~mm}^{2}$ and the duration of exposure at the time of treatment should not be more then few hours. ${ }^{4}$ Cvek pulpotomy which is a technique of partial pulpotomy, is considered as treatment of choice when pulpal exposure is more than $1 \mathrm{~mm}^{2}$ and time lapse is long between the incident and examination. ${ }^{4}$

Until 1983 calcium hydroxide mixture was used as a dressing material to initiate reparative dentin formation by 
controlling infection and stimulating the pulp healing process. ${ }^{4}$ Over the past few decades, MTA has taken the endodontic world by its storm. It improves the healing capacity of pulp tissue as it provides good ability to seal, biocompatibility, low cytotoxicity and also induces odontoblast for forming a dentinal bridge barrier. Hence MTA is considered now as a gold standard pulp dressing material. ${ }^{5}$

Esthetic treatment options for coronal tooth fractures involves ceramic (laminated veneers, full crown) or composite restorations and re-attachment of fractured tooth fragment. ${ }^{6}$

Fragment re-attachment is the finest option if the broken tooth fragment is available and in a condition that it can be used with proper occlusion, esthetics and good prognosis. ${ }^{6}$ In 1964, Chosak and Eidelman was the first who published this technique. ${ }^{7}$ Tooth fragment re-attachment provides a more conservative, esthetic and cost effective restorative approach to restore tooth function and esthetics similar to natural teeth, thus resulting a positive psychological response in the patient. ${ }^{8}$

Throughout the literature, different preparation techniques have been described to increase the retention of broken tooth fragment mechanically such as placement of a circumferential bevel, enamel groove, external chamfer, overcontour, dentinal groove and different types of adhesive materials. ${ }^{9}$

Reis have concluded that fractures restored with no further preparation in broken fragment or in effected tooth have only $37 \%$ fracture resistance, while $60 \%$ fracture resistance is increased by introducing a buccal chamfer. Placement of internal groove and bonding with over contouring further increased the strength of intact tooth fracture upto $90 \%$ and $97 \%$ respectively. ${ }^{9}$

Presented case describes the treatment of complicated crown fracture with Cvek's pulpotomy using MTA followed by re-attachment of broken fragment.

\section{CASE REPORT}

A female patient of 12 years old is presented to Pediatric dental department at Pakistan Institute of Medical Sciences, Islamabad with the chief complaint of broken upper front tooth due to trauma one hour ago. She brought the broken fragment kept in saline. Medically she was fit and well with no known medical history. The intraoral examination revealed middle third horizontal coronal fracture of upper left central incisor with the pulp exposure of more than $1 \mathrm{~mm}^{2}$. Extraoral examination showed a small laceration on the ipsilateral side of lower lip (fig a,b).

Diagnosis was made after doing clinical tests and radiographic examination. Three radiographs at different vertical angulations were done to rule out any root fracture. Radiographic examination revealed complicated oblique crown fracture of involved tooth involving the pulp. There was a slightly widened lamina dura of the involved tooth, without any root fracture or any periapical radiolucency. (fig c)

Cold test was done initially on the sound adjacent tooth (upper right central incisor) to evaluate the normal response
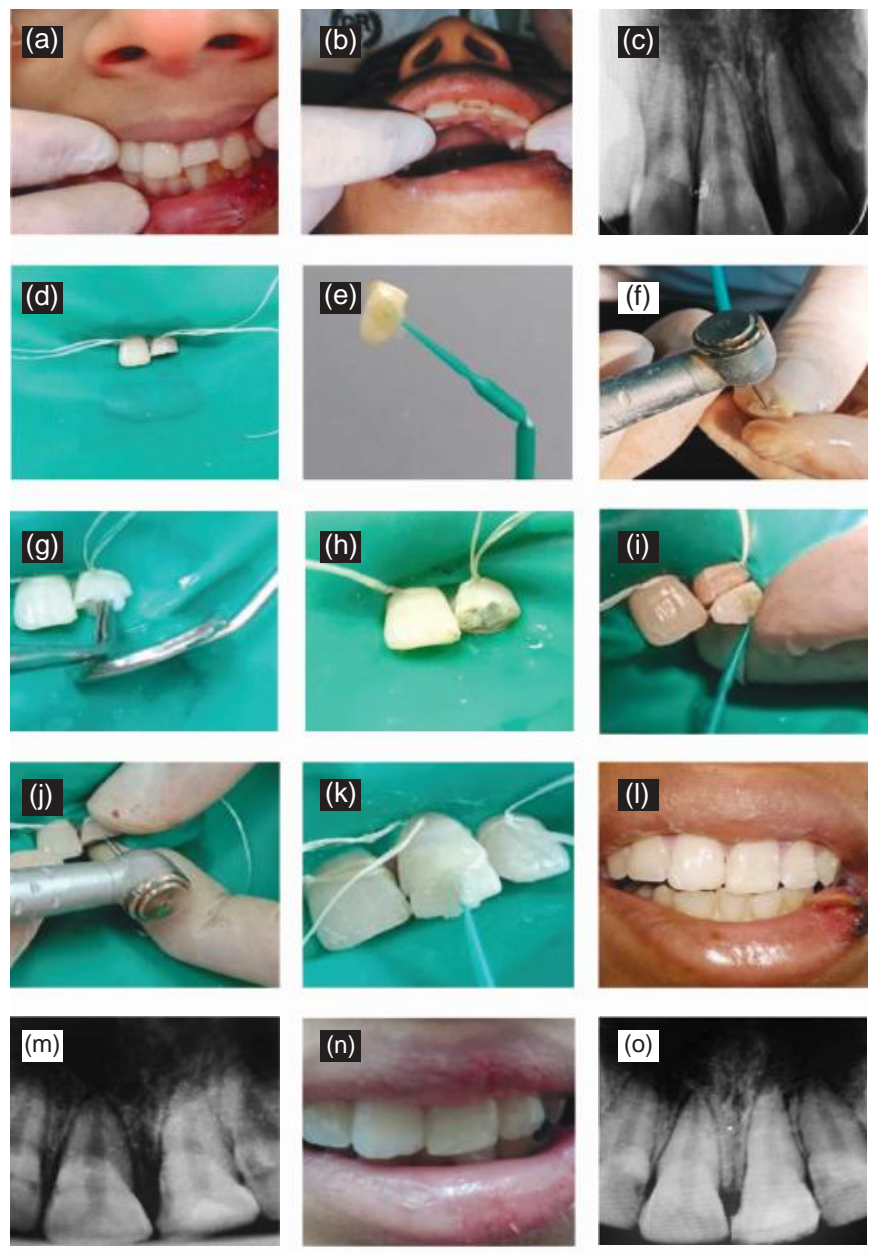

Figure (a) preoperative intraoral picture showing fractured left maxillary central incisor and lower lip laceration (b) fractured tooth showing pulp exposure (c) Periapical radiograph showing widened lamina dura $(d)$ Rubber dam application (e) holding tooth for preparation to prevent any tooth movement $(f)$ internal groove preparation in the fracture segment to increase retention. $(g)$ hemorrhage control by using moist cotton pellet ( $h$ ) MTA has been placed after attaining hemostasis. (i) stabilization of fracture segment on a micro brush to facilitate handling. $(J)$ repositioning and bonding of the fracture segment via flowable composite. $(k)$ restoration done via packable

composite.(l)postoperative clinical picture after finishing and polishing. $(m)$ immediate postoperative radiograph.(n) follow up after 1 week. (0) last picture showing postoperative radiograph after 1 year.

by using ethyl chloride.Again it was done on upper left central incisor (traumatized tooth) which showed no response, because of the pulp shock. The tooth was not mobile but was slightly tender to percussion because of the associated 
subluxation and PDL injury.

Lateral soft tissue radiograph of lower lip was done in order to rule out any foreign body. No foreign body was present upon radiographic evaluation.

After clinical and radiographic evaluation, the definitive diagnosis cannot be done as it was traumatized 1hour ago. The decision was made to perform cvek (partial) pulpotomy with MTA (ProRoot, Dentsply) as pulp exposure was more than $1 \mathrm{~mm}^{2}$, followed by fragment reattachment of traumatized tooth.. Broken tooth fragment was assessed intraorally for proper occlusion.

After taking an informed consent, topical anesthesia (benzocaine $20 \%$, keystone USA) was applied after drying the oral mucosa. Local maxillary infiltration was done with $2 \%$ lidocaine $(1: 100,000)$.

To prevent salivary contamination, operating field was isolated using rubber dam. (fig d) Tooth was washed with copious amount of saline. By holding the tooth, $2 \mathrm{~mm}$ coronal pulp tissue, below the level of pulp exposure was gently removed using sterile round bur mounted on high speed handpiece with continuous saline irrigation (fig e).

Bleeding was controlled by placing sterile moistened cotton pellet. (fig f) after attaining hemostasis within 3 minutes, MTA (ProRoot, Dentsply) powder was dispensed on a glass slab and mixed with distilled water according to the manufacturer's recommendation and placed over the exposed pulp without any pressure. Afterward, a Resin modified Glass Ionomer Cement (FUJI IX, GC Corporation, Tokyo, Japan) was applied as a base material to seal the cavity.

Prior to re-attachment procedure sharp margins of the tooth and the broken fragment were smoothened. Using small sterile \#2 round bur (R40004G, Coltene) internal groove was made on a broken fragment to increase retention (fig f). Broken fragment was then secured with the tip of bonding brush in order to facilitate handling. It is then etched by using 37\% phosphoric acid (META BIOMED CO.Lt) for $30 \mathrm{sec}$ followed by washing for $20 \mathrm{sec}$ and drying with a moist cotton pellet. Dentine bonding agent (Meta P \& Bond, META BIOMED) then applied over the etched surface and light curing was not done at this point.

Likewise, fractured residual tooth surface also treated with 37\% phosphoric acid etchant (META BIOMED CO.Lt) then washed and same dentine bonding agent (Meta P \& Bond, META BIOMED) was applied to it. Flowable composite resin (Filtek Z350 XT, USA) was then applied to both broken fragment and fractured tooth surface. Fragment was repositioned properly on the tooth. Excessive resin was wiped off after establishing the appropriate position and light cured for $40 \mathrm{sec}$ on both labial and lingual surfaces. (fig g).
After assuring proper stability of the fragment, 'double chamfer' margin of $1 \mathrm{~mm}$ was given using a round end tapered bur(dentsply) above and below the fracture line of the tooth. Permanent composite restoration (3M ESPE, Z250,USA) was done to restore the tooth. (fig i) It was then finished and polished. Gross contouring and finishing was initially done with tapered round ended finishing bur(DENTSPLY/Caulk). Intermediate contouring and finishing was done with soflex discs ( $3 \mathrm{M}$, ESPE) then final polishing was done with diamond polishing paste (Kerr).

Rubber dam was removed and occlusion was checked carefully by using an articulating paper. Post-operative instructions to abstain from applying heavy occlusal forces on this tooth was given and patient was motivated to practice good oral hygiene. Before dismissing the patient, PA radiograph of the treated tooth was done for comparison in the follow-up appointments (fig j).

Careful clinical and radiographic examinations were done after 1 week, 3 weeks and 6 weeks in order to check the vitality of the tooth and root growth. Periapical radiographs showed no pathological changes at 1 week, 3 weeks and 6weeks.Cold test was done at each follow-up appointment which showed negative response at 1 week postoperatively and showed positive response at 3 week and 6 week of follow-up examination. (fig $\mathrm{n}$ ) Percussion test was also done at each followup visit that showed negative response.

\section{DISCUSSION}

Complicated crown fracture are the fractures involving both dentine and enamel with pulp exposure. 18-20\% of all the traumatic injuries involves these type of fractures. ${ }^{2}$ Such fractures should be managed as early as possible to prevent pulp necrosis. To keep the pulp vital, in fractures involving pulp exposure of upto $4 \mathrm{~mm}$, Partial vital pulpotomy is the treatment of choice. $96 \%$ success rate is reported by Cvek in such cases with long follow up period. ${ }^{10}$

Calcium hydroxide was previously used in vital pulpotomy. MTA is now the material of choice with reparation mechanism is similar to calcium hydroxide as it provides better long term seal and produce more dentinal bridging in relatively shorter period of time with less pulp tissue inflammation. ${ }^{10}$

Secondly, in the current case the method used to reattach the fracture segment involves preparation of the internal groove in the fractured fragment and double chamfer formation on the crown portion $1 \mathrm{~mm}$ above and below fracture line to improve strength and retention.

One year clinical and radiographic follow up was done. Clinical results at the end of follow up showed adequate aesthetic and functional results of fragment re-attachment 
technique with no pain, sensitivity, pathological pulpal changes and change of tooth color. Radiographic examination also showed no pathological periapical / peri-radicular changes, root resorption or calcification of canals.

\section{CONFLICT OF INTEREST}

None to declare

\section{REFERENCES}

1. J. O. Andreasen, "Etiology and pathogenesis of traumatic dental injuries: a clinical study of 1,298 cases," Scand J Dent Res. 1970; 78: 329-42.

https://doi.org/10.1111/j.1600-0722.1970.tb02080.x

2. De Blanco LP. Treatment of crown fractures with pulp exposure. Oral Surg Oral Med Oral Pathol Oral Radiol Endod. 1996;82:564-8. https://doi.org/10.1016/S1079-2104(96)80204-6

3. Olsburgh S, Jacoby T, \& Krejci I. Crown fractures in the permanent dentition: Pulpal and restorative considerations Dent Traumatol. 2002;18:103-15.

https://doi.org/10.1034/j.1600-9657.2002.00004.x

4. Cvek M. Endodontic treatment of traumatized teeth. In: Andreasen JO, editor. Traumatic injuries to the teeth. 2nd ed. Copenhagen: Blackwell Munksgaard; 2003;321-83.
5. Ford TR, Torabinejad M, Abedi HR, Bakland LK, Kariyawasam SP. Using mineral trioxide aggregate as a pulp capping material. J Am Dent Assoc. 1996;127:1491-94.

https://doi.org/10.14219/jada.archive.1996.0058

6. Alvares I, Sensi LG, Araujo EM Jr, \& Araujo E. Silicone index: An alternative approach for tooth fragment reattachment. J Esthetic Restorative Dentist. 2007;19:240-46.

https://doi.org/10.1111/j.1708-8240.2007.00110.x

7. CMC Taguchi, JK Bernardon, G Zimmermann, and LN Baratieri. Tooth Fragment Reattachment: A Case Report. Operative Dentistry: 2015;40;227-34.

https://doi.org/10.2341/14-034-T

8. Badami V, \& Reddy SK. Treatment of complicated crown-root fracture in a single visit by means of rebonding. J Am Dent Assoc. 2011;142:646-50

https://doi.org/10.14219/jada.archive.2011.0246

9. Reis A, Francci C, Loguercio AD, et al. Re-attachment of anterior fractured teeth: fracture strength using different techniques. Oper Dent 2001;26:287-94.

10. M. Cvek, "A clinical report on partial pulpotomy and capping with calcium hydroxide in permanent incisors with complicated crown fracture," J Endod. 1978;232-37.

https://doi.org/10.1016/S0099-2399(78)80153-8 